PHYSICAL REVIEW D 94, 126016 (2016)

\title{
Dynamical angled brane
}

\author{
Kei-ichi Maeda \\ Department of Physics, Waseda University, Okubo 3-4-1, Shinjuku, Tokyo 169-8555, Japan
}

Kunihito Uzawa

Department of Physics, School of Science and Technology, Kwansei Gakuin University, Sanda, Hyogo 669-1337, Japan

(Received 7 March 2016; published 27 December 2016)

\begin{abstract}
We discuss the dynamical $\mathrm{D} p$-brane solutions describing any number of $\mathrm{D} p$ branes whose relative orientations are given by certain $\mathrm{SU}(2)$ rotations. These are the generalization of the static angled $\mathrm{D} p$-brane solutions. We study the collision of the dynamical D3 brane with angles in type-II string theory, and show that the particular orientation of the smeared D3-brane configuration can provide an example of colliding branes if they have the same charges. Otherwise a singularity appears before D3 branes collide.
\end{abstract}

DOI: 10.1103/PhysRevD.94.126016

\section{INTRODUCTION}

The time-dependent brane solutions in string theory were introduced in [1-29] and have been widely used ever since. But some aspects of the physical properties such as dynamical $p$ brane oriented at angles have remained slightly unclear.

The angled $\mathrm{D} p$-brane solutions were discovered by [30], following the recognition of several roles of D-brane configurations in string theory $[31,32,34]$. The classical solution on ten-dimensional spacetime that should be obtained to introduce oriented angles in string theories was studied from several points of view in $[21,30,34,35]$. The authors have discussed the link between the supersymmetric configurations, intersection of the brane, and rotation angles. The other completely consistent computations for angled D branes were performed in [31-33]. The articles $[34,36,37]$ give a thorough review of much of what was known in the late 1990s.

These results are more transparent if the dynamical $\mathrm{D} p$ brane solution in string theory is discussed in terms of supergravity theory, aiming to reduce everything to ordinary higher-dimensional general relativity. In the present paper, we construct new solutions with nontrivial angles in string theory and study the dynamical behavior that one would expect of a string theory with the time-dependent fields and branes.

We start by describing the appropriate ansatz of the fields and metric in string theory, and find the solution of the dynamical D2 brane with angle in Sec. II A. The solution is straightforwardly generalized from the static angled D2brane background. This is a good illustration, and also an important example in order to understand the orientation of a particular type of brane configuration, which is the socalled angled D-brane system [30-32].

Using the T-duality map between the type IIA and IIB string theories, we can obtain the dynamical angled
D3- and D4-brane solutions in terms of dynamical D2 branes, which are discussed in Appendix. We summarize the general dynamical $N \mathrm{D} p$-brane system with angles in Sec. II B, provide their properties in Sec. III and discuss the collision of two D3 branes in the presence of angles in Sec. IV. Finally, Sec. V is devoted to a summary and discussion.

\section{DYNAMICAL D $p$-BRANE SYSTEM WITH ANGLES}

\section{A. D2-brane system with angle}

In this subsection, we consider dynamical D2 branes oriented at some angles in ten dimensions. The tendimensional spacetime metric depends on time as well as the rotation angles which describe the orientations of various D2 branes.

\section{Basic equations}

The action for the D2-brane system in the Einstein frame is written as

$S=\frac{1}{2 \kappa^{2}} \int\left(R * \mathbf{1}-\frac{1}{2} * d \phi \wedge d \phi-\frac{1}{2 \cdot 4 !} \mathrm{e}^{\phi / 2} * F_{(4)} \wedge F_{(4)}\right)$,

where $\kappa^{2}$ is the ten-dimensional gravitational constant, $*$ is the Hodge dual operator in the ten-dimensional spacetime, and $F_{(4)}$ is the 4-form field strength. We assume that the 4-form $F_{(4)}$ in the action Eq. (1) is now given by

$$
F_{(4)}=d C_{(3)},
$$

where $C_{(3)}$ is the 3-form gauge field. After variations with respect to the metric, the scalar field, and the gauge field, we obtain the field equations, 


$$
\begin{gathered}
R_{M N}=\frac{1}{2} \partial_{M} \phi \partial_{N} \phi \\
+\frac{1}{2 \cdot 4 !} \mathrm{e}^{\phi / 2}\left(2 F_{M A B C} F_{N}^{A B C}-\frac{3}{8} g_{M N} F_{(4)}^{2}\right), \\
d * d \phi=\frac{1}{4 \cdot 4 !} \mathrm{e}^{\phi / 2} * F_{(4)} \wedge F_{(4)}, \\
d\left(\mathrm{e}^{\phi / 2} * F_{(4)}\right)=0 .
\end{gathered}
$$

\section{Single D2-brane solution with angle}

First we consider a single D2-brane solution. The geometry of dynamical D2-brane configuration in tendimensional spacetime is assumed to be

$$
\begin{gathered}
d s^{2}=h^{-5 / 8}(t, z)\left(-d t^{2}+\gamma_{i j} d y^{i} d y^{j}\right) \\
+h^{3 / 8}(t, z) u_{a b}(z) d z^{a} d z^{b} \\
\gamma_{i j} d y^{i} d y^{j}=\delta_{i j} d y^{i} d y^{j}+f(t, z)\left[\left(\cos \theta d y^{1}-\sin \theta d y^{2}\right)^{2}\right. \\
\left.+\left(\cos \theta d y^{3}+\sin \theta d y^{4}\right)^{2}\right] \\
h(t, z)=1+f(t, z),
\end{gathered}
$$

where $d s^{2}$ is the line element in the Einstein frame in ten dimensions, $\delta_{i j}$ is the four-dimensional flat Euclidean metric, and $u_{a b}$ is the metric of the five-dimensional space $\mathrm{Z}$ depending only on the five-dimensional coordinates $z^{a}$. The dilaton field and the gauge field strengths are assumed to be

$$
\begin{gathered}
\mathrm{e}^{\phi}=h^{1 / 2}, \\
F_{(4)}=d\left[1-h^{-1}(t, z)\right] \wedge d t \wedge\left(-\sin ^{2} \theta d y^{1} \wedge d y^{3}\right. \\
+\sin \theta \cos \theta d y^{1} \wedge d y^{4} \\
\left.-\sin \theta \cos \theta d y^{2} \wedge d y^{3}+\cos ^{2} \theta d y^{2} \wedge d y^{4}\right) .
\end{gathered}
$$

We first solve the equation for the gauge field strength $F_{(4)}$. Substituting the above ansatz for the fields and the metric form into Eq. (3c), we find

$$
\partial_{t} \partial_{a} h=0, \quad \Delta_{\mathrm{Z}} h=0 .
$$

The function $h$ is given by

$$
h(t, z)=h_{0}(t)+h_{1}(z) .
$$

Then we solve the equation for the scalar field, Eq. (3b). Using the assumptions (4) and (5b), we have

$$
h^{-3 / 8}\left(\partial_{t}^{2} h-h^{-1} \Delta_{Z} h\right)=0,
$$

where $\Delta_{\mathrm{Z}}$ is the Laplace operator on Z. Combining Eq. (8) with Eqs. (6) and (7), we find

$$
\partial_{t}^{2} h_{0}=0, \quad \Delta_{\mathrm{Z}} h_{1}=0 .
$$

Finally we analyze the Einstein equations (3a). Using the ansatz (4) and (5), the Einstein equations become

$$
\begin{gathered}
\frac{11}{16} h^{-1} \partial_{t}^{2} h+\frac{5}{16} h^{-2} \Delta_{\mathrm{Z}} h=0, \\
h^{-1} \partial_{t} \partial_{a} h=0, \\
\frac{5}{16} h^{-1} \gamma_{i j}\left(\partial_{t}^{2} h-h^{-1} \Delta_{\mathrm{Z}} h\right)+\frac{1}{2} h^{-1} \Delta_{\mathrm{Z}} \gamma_{i j}=0, \\
R_{a b}(\mathrm{Z})+\frac{3}{16} u_{a b}\left(\partial_{t}^{2} h-h^{-1} \Delta_{\mathrm{Z}} h\right)=0,
\end{gathered}
$$

where $R_{a b}(\mathrm{Z})$ is the Ricci tensor of the five-dimensional space $\mathrm{Z}$. Hence, the field equations reduce to

$$
\begin{gathered}
R_{a b}(\mathrm{Z})=0, \\
h(t, z)=h_{0}(t)+h_{1}(z), \quad \partial_{t}^{2} h_{0}=0, \\
\Delta_{\mathrm{Z}} h_{1}=0, \quad \Delta_{\mathrm{Z}} f=0 .
\end{gathered}
$$

This is the exact solution of the present system for any given Ricci flat metric $u_{a b}$.

As an example, we set

$$
u_{a b}=\delta_{a b},
$$

where $\delta_{a b}$ is the five-dimensional Euclidean metric. Then we find an exact solution for a single brane solution,

$$
h_{0}(t)=c_{0} t+c_{1}, \quad h_{1}(z)=c_{2}+\frac{Q_{1}}{\left|z^{a}-z_{(1)}^{a}\right|^{3}},
$$

where $c_{0}, c_{1}, c_{2}, Q_{1}$, and $z_{(1)}^{a}$ are constants. $Q_{1}$ is the charge of the D2 brane, and $z_{(1)}^{a}$ is the position of the D2 brane. The mass of the brane is given by $M_{1}=\left|Q_{1}\right|$. If, however, some spatial dimensions of $\mathrm{Z}$ space are smeared by the $\mathrm{D} 2$ branes, we find

$$
h_{0}(t)=c_{0} t+c_{1}, \quad h_{1}(z)=c_{2}+\frac{Q_{1}}{\left|z^{a}-z_{(1)}^{a}\right|^{3-d_{z}}},
$$

where $d_{\mathrm{Z}}(<5)$ is the smeared dimensions. For the case of $d_{\mathrm{Z}}=3$, we have to replace $h_{1}$ with

$$
h_{1}(z)=c_{2}+Q_{1} \ln \left|z^{a}-z_{(1)}^{a}\right| .
$$

Although the above exact solution [Eqs. (4) and (5)] includes the orientation angle $\theta$ in the solution, it is equivalent to a single brane solution without an angle. In fact we find that the above solution is reduced to a single D2-brane solution without an angle if we rotate the $y_{1}-y_{2}$ and $y_{3}-y_{4}$ planes by the angles $\theta$ and $-\theta$, respectively, as 


$$
\begin{aligned}
& {\left[\begin{array}{l}
y^{1 \prime} \\
y^{2 \prime}
\end{array}\right]=\left(\begin{array}{cc}
\cos \theta & -\sin \theta \\
\sin \theta & \cos \theta
\end{array}\right)\left[\begin{array}{l}
y^{1} \\
y^{2}
\end{array}\right],} \\
& {\left[\begin{array}{l}
y^{3 \prime} \\
y^{4 \prime}
\end{array}\right]=\left(\begin{array}{cc}
\cos \theta & \sin \theta \\
-\sin \theta & \cos \theta
\end{array}\right)\left[\begin{array}{l}
y^{3} \\
y^{4}
\end{array}\right] .}
\end{aligned}
$$

This solution is nothing but that without an angle. However this description is important to construct the $N$-brane system with different angles as we show it below. Note that if all $N$ D2 branes are parallel each other, we find the exact solution for $N \mathrm{D} 2$ branes with the same angle $\theta$ as Eq. (4) with Eq. (7) and

$$
h_{0}(t)=c_{0} t+c_{1}, \quad h_{1}(z)=c_{2}+\sum_{\alpha=1}^{N} \frac{Q_{\alpha}}{\left|z^{a}-z_{(\alpha)}^{a}\right|^{3-d_{Z}}},
$$

where $c_{0}, c_{1}, c_{2}, Q_{\alpha}$, and $z_{(\alpha)}^{a}$ are constants and $d_{\mathrm{Z}}(=0, \ldots, 4)$ is the smeared dimensions. $Q_{\alpha}$ and $z_{(\alpha)}^{a}$ denote the charges of branes and their positions, respectively. In the case of $d_{\mathrm{Z}}=3$, the power function must be replaced by $\ln \left|z^{a}-z_{(\alpha)}^{a}\right|$.

\section{Two D2-brane system with angles}

Next we construct the exact solutions of two intersecting D2 branes with nontrivial angle. The ten-dimensional metric, scalar field and single 3-form potential are assumed to be

$$
\begin{gathered}
d s^{2}=h^{-5 / 8}(t, z)\left(-d t^{2}+\gamma_{i j} d y^{i} d y^{j}\right) \\
+h^{3 / 8}(t, z) u_{a b}(z) d z^{a} d z^{b}, \\
\gamma_{i j} d y^{i} d y^{j}=\delta_{i j} d y^{i} d y^{j}+f_{(1)}\left[\left(\cos \theta_{1} d y^{1}-\sin \theta_{1} d y^{2}\right)^{2}\right. \\
\left.+\left(\cos \theta_{1} d y^{3}+\sin \theta_{1} d y^{4}\right)^{2}\right] \\
+f_{(2)}\left[\left(\cos \theta_{2} d y^{1}-\sin \theta_{2} d y^{2}\right)^{2}\right. \\
\left.+\left(\cos \theta_{2} d y^{3}+\sin \theta_{2} d y^{4}\right)^{2}\right], \\
\quad h(t, z)=1+f_{(1)}(t, z)+f_{(2)}(t, z) \\
\quad+f_{(1)}(t, z) f_{(2)}(t, z) \sin ^{2} \theta, \\
\quad \mathrm{e}^{\phi}=h^{1 / 2}, \\
C_{(3)}=h^{-1}(t, z) d t \wedge\left[-\left\{f_{(1)} \sin ^{2} \theta_{1}+f_{(2)} \sin ^{2} \theta_{2}\right.\right. \\
\left.+f_{(1)} f_{(2)} \sin ^{2}\left(\theta_{1}-\theta_{2}\right)\right\} d y^{1} \wedge d y^{3}+\left(f_{(1)} \sin \theta_{1} \cos \theta_{1}\right. \\
\left.+f_{(2)} \sin \theta_{2} \cos \theta_{2}\right)\left(d y^{1} \wedge d y^{4}-d y^{2} \wedge d y^{3}\right) \\
+\left\{f_{(1)} \cos ^{2} \theta_{1}+f_{(2)} \cos ^{2} \theta_{2}\right. \\
\left.\left.+f_{(1)} f_{(2)} \cos ^{2}\left(\theta_{1}-\theta_{2}\right)\right\} d y^{2} \wedge d y^{4}\right],
\end{gathered}
$$

where $\theta=\theta_{2}-\theta_{1}$. The notable property with the nontrivial angle appears in the nonlinear interaction term in Eq. (18c).
In the following, we consider the two D2-brane system with the different rotation angles $\left(\theta_{1}, \theta_{2}\right)$ where $\theta_{1} \neq \theta_{2}$. We assume $\left(\theta_{1}, \theta_{2}\right)=(0, \theta)(\theta \neq 0)$ without loss of generality, because we can always set one angle to be 0 by an appropriate rotation of $y^{i}$-coordinates.

In terms of the ansatz for fields and the metric, the gauge field equation (3c) gives

$$
\begin{aligned}
\partial_{t} \partial_{a} f_{(1)} & =0, & \Delta_{Z} f_{(1)} & =0, \\
\partial_{t} \partial_{a} f_{(2)} & =0, & \Delta_{Z} f_{(2)} & =0 .
\end{aligned}
$$

From the equation of scalar field, we find

$$
\begin{gathered}
h^{-3 / 8}\left[\partial_{t}^{2} h-h^{-1}\left\{\left(1+f_{(2)} \sin ^{2} \theta\right) \Delta_{\mathrm{Z}} f_{(1)}\right.\right. \\
\left.\left.+\left(1+f_{(1)} \sin ^{2} \theta\right) \Delta_{\mathrm{Z}} f_{(2)}\right\}\right]=0 .
\end{gathered}
$$

Under our ansatz, the Einstein equations become

$$
\begin{aligned}
& \frac{11}{16} h^{-1} \partial_{t}^{2} h+\frac{5}{16} h^{-2}\left[\left(1+f_{(2)} \sin ^{2} \theta\right) \Delta_{\mathrm{Z}} f_{(1)}\right. \\
& \left.\quad+\left(1+f_{(1)} \sin ^{2} \theta\right) \Delta_{\mathrm{Z}} f_{(2)}\right]=0
\end{aligned}
$$

$h^{-1}\left[\left(1+f_{2} \sin ^{2} \theta\right) \partial_{t} \partial_{a} f_{1}+\left(1+f_{(1)} \sin ^{2} \theta\right) \partial_{t} \partial_{a} f_{(2)}\right]=0$,

$$
\begin{aligned}
& \frac{5}{16} h^{-1} \gamma_{i j}\left[\partial_{t}^{2} h-h^{-1}\left\{\left(1+f_{(2)} \sin ^{2} \theta\right) \Delta_{\mathrm{Z}} f_{(1)}\right.\right. \\
& \left.\left.+\left(1+f_{(1)} \sin ^{2} \theta\right) \Delta_{\mathrm{Z}} f_{(2)}\right\}\right]+\frac{1}{2} h^{-1} \Delta_{\mathrm{Z}} \gamma_{i j}=0, \\
& R_{a b}(\mathrm{Z})+\frac{3}{16} u_{a b}\left[\partial_{t}^{2} h-h^{-1}\left\{\left(1+f_{(2)} \sin ^{2} \theta\right) \Delta_{\mathrm{Z}} f_{(1)}\right.\right. \\
& \left.\left.\quad+\left(1+f_{(1)} \sin ^{2} \theta\right) \Delta_{\mathrm{Z}} f_{(2)}\right\}\right]=0,
\end{aligned}
$$

where $R_{a b}(\mathrm{Z})$ is the Ricci tensor of the $\mathrm{Z}$ space. Then, the field equations reduce to

$$
R_{a b}(\mathrm{Z})=0,
$$

$$
\begin{aligned}
& f_{(1)}(t, z)=\tilde{f}_{(1)}(t)+\bar{f}_{(1)}(z), \\
& f_{(2)}(t, z)=\tilde{f}_{(2)}(t)+\bar{f}_{(2)}(z),
\end{aligned}
$$

$\partial_{t}^{2} \tilde{f}_{(1)}=0, \quad \partial_{t}^{2} \tilde{f}_{(2)}=0, \quad \partial_{t} \tilde{f}_{(1)} \partial_{t} \tilde{f}_{(2)}=0$,

$\Delta_{\mathrm{Z}} \bar{f}_{(1)}=0, \quad \Delta_{\mathrm{Z}} \bar{f}_{(2)}=0$.

For a given Ricci flat $\mathrm{Z}$ space, we can obtain the exact solution for the two D2-brane system with different angles by solving the above equation (22c). As a result, at least one of $\tilde{f}_{(1)}$ and $\tilde{f}_{(2)}$ must be constant and then only one brane can be time dependent, i.e., for $\alpha=1$ or 2 , 


$$
\tilde{f}_{(\alpha)}=c_{(\alpha) 0} t+c_{(\alpha) 1},
$$

where $c_{(\alpha) 0}, c_{(\alpha) 1}$ are constant, and the other $\tilde{f}_{(\beta)}=c_{(\beta) 1}$ $(\beta \neq \alpha)$ are constant. Following the same procedure as the case of the two D2-brane, we can easily generalize the above solution to the $N$ D2-brane system with different angles $\theta_{\alpha}(\alpha=1, \ldots, N)$.

\section{N D2-brane system with angles}

Now we present the solution of field equations for the $N$ D2-brane system with different angles $\theta_{\alpha}(\alpha=1, \ldots, N)$.

The ten-dimensional metric $[30,34]$ is given by

$$
\begin{gathered}
d s^{2}=h^{-5 / 8}\left[-d t^{2}+\gamma_{i j} d y^{i} d y^{j}+h u_{a b}(\mathrm{Z}) d z^{a} d z^{b}\right], \\
\gamma_{i j} d y^{i} d y^{j}=\delta_{i j} d y^{i} d y^{j}+\sum_{\alpha=1}^{N} f_{(\alpha)}\left[\left\{\left(R_{(\alpha)}\right)^{1}{ }_{i} d y^{i}\right\}^{2}\right. \\
\left.+\left\{\left(R_{(\alpha)}\right)^{3}{ }_{j} d y^{j}\right\}^{2}\right] \\
h(t, z)=1+f(t, z) .
\end{gathered}
$$

The metric (24) denotes the $N$ D2-brane system such that each brane first lying in the $\left(y^{2}, y^{4}\right)$ plane rotates by an angle $\theta_{\alpha}(\alpha=1, \ldots, N)$ in the $\left(y^{1}, y^{2}\right)$ and $\left(y^{3}, y^{4}\right)$ planes as $(\xi, \eta) \rightarrow\left(\mathrm{e}^{i \theta_{\alpha}} \xi, \mathrm{e}^{-i \theta_{\alpha}} \eta\right)$ where $\xi=y^{1}+i y^{2}$ and $\eta=y^{3}+i y^{4}$, the rotation of which belongs to the $\mathrm{SU}(2)$ group. The function $h$ and the rotation matrix $R_{(\alpha)}$ associated with the $\alpha$ th D2-brane are given by

$$
\begin{gathered}
f(t, z)=\sum_{\alpha=1}^{N} f_{(\alpha)}+\sum_{\alpha<\beta}^{N} f_{(\alpha)} f_{(\beta)} \sin ^{2}\left(\theta_{\alpha}-\theta_{\beta}\right), \\
R_{(\alpha)}=\left(\begin{array}{cccc}
\cos \theta_{\alpha} & -\sin \theta_{\alpha} & 0 \\
\sin \theta_{\alpha} & \cos \theta_{\alpha} & \cos \theta_{\alpha} & \sin \theta_{\alpha} \\
0 & -\sin \theta_{\alpha} & \cos \theta_{\alpha}
\end{array}\right),
\end{gathered}
$$

where $R_{(\alpha)}(\alpha=1, \ldots, N)$ are $\mathrm{SO}(4)(\cong \mathrm{SU}(2))$ matrices that correspond to the rotation of D2 branes. The scalar field $\phi$ and the 3 -form gauge field $C_{(3)}$ are given by

$$
\begin{aligned}
C_{(3)}= & h^{-1} d t \wedge\left\{\sum_{\alpha=1}^{N} f_{(\alpha)}\left(R_{(\alpha)}\right)_{i}^{2} d y^{i} \wedge\left(R_{(\alpha)}\right)_{j}^{4} d y^{j}\right. \\
& \left.-\sum_{a<b}^{N} f_{(\alpha)} f_{(\beta)} \sin ^{2}\left(\theta_{\alpha}-\theta_{\beta}\right)\left(d y^{1} \wedge d y^{3}-d y^{2} \wedge d y^{4}\right)\right\},
\end{aligned}
$$

$$
\mathrm{e}^{2 \phi}=h^{1 / 2} .
$$

The assumption for fields is a straightforward generalization of the case of a static D2-brane system with a certain SU(2) angle, in the type-IIA low energy effective string theory $[30,34]$. From the field equations, the ten-dimensional metric (24) has to obey

$$
\begin{gathered}
R_{a b}(\mathrm{Z})=0, \\
f_{(\alpha)}(t, z)=\tilde{f}_{(\alpha)}(t)+\bar{f}_{(\alpha)}(z), \\
\partial_{t}^{2} \tilde{f}_{(\alpha)}=0, \quad \partial_{t} \tilde{f}_{(\alpha)} \partial_{t} \tilde{f}_{(\beta)}=0, \quad(\alpha \neq \beta) \\
\Delta_{\mathrm{Z}} \bar{f}_{(\alpha)}=0 .
\end{gathered}
$$

This gives the exact solution for a $N$ D2-brane system with different angles for giving Ricci flat $\mathrm{Z}$ space. As the two brane system, only one brane can be time dependent,

$$
\tilde{f}_{(\alpha)}=c_{(\alpha) 0} t+c_{(\alpha) 1},
$$

where $c_{(\alpha) 0}, c_{(\alpha) 1}$ are constant, and the other $\tilde{f}_{(\beta)}=c_{(\beta) 1}$ $(\beta \neq \alpha)$ are constant. The interesting property for the case with the nontrivial angle is the nonlinear interaction term found in Eq. (25a).

For the case of $u_{a b}=\delta_{a b}$, where $\delta_{a b}$ is the five-dimensional flat Euclidean metric, the functions $\bar{f}_{(\alpha)}$ are given by harmonic functions, which are associated with D2 branes located at $z^{a}=z_{(\alpha)}^{a}$,

$$
\bar{f}_{(\alpha)}(z)=\bar{c}_{\alpha}+\frac{Q_{\alpha}}{\left|z^{a}-z_{(\alpha)}^{a}\right|^{3-d_{\mathrm{Z}}}},
$$

where $c_{\alpha}, Q_{\alpha}$ and $z_{(\alpha)}^{a}$ are constants and $d_{\mathrm{Z}}(=0, \ldots, 4)$ is the smeared dimensions in the Z space. In the case of $d_{\mathrm{Z}}=3$, the power function must be replaced by $\ln \left|z^{a}-z_{(\alpha)}^{a}\right|$.

\section{B. Dp-brane system with angles}

In order to find the configurations of a higherdimensional $\mathrm{D} p$-brane system with angles $(p>2)$, we use a T duality. In Appendix, we present the explicit procedure for the D3- and D4-brane system with angles. For the case of $p \geq 5$, we can repeat the same procedure. Here we summarize the solution of the $N \mathrm{D} p$-brane system with different $\mathrm{SU}(2)$ angles as follows.

The metric is given by

$$
\begin{aligned}
d s^{2}= & h^{\frac{p-7}{8}}\left[-d t^{2}+\gamma_{i j} d y^{i} d y^{j}+\delta_{m n} d \zeta^{m} d \zeta^{n}\right. \\
& \left.+h u_{a b}(\mathrm{Z}) d z^{a} d z^{b}\right]
\end{aligned}
$$

with 


$$
\begin{gathered}
\gamma_{i j} d y^{i} d y^{j}=\delta_{i j} d y^{i} d y^{j}+\sum_{\alpha=1}^{N} f_{(\alpha)}\left[\left\{\left(R_{(\alpha)}\right)^{1} d y^{i}\right\}^{2}\right. \\
\left.+\left\{\left(R_{(\alpha)}\right)^{3} d y^{j}\right\}^{2}\right], \\
h(t, z)=1+f(t, z) .
\end{gathered}
$$

The number of the dimensions of the $\zeta^{m}$-space is $p-2$, assuming $p \geq 2$. The functions $f(t, z)$ and $f_{(\alpha)}(t, z)$ in the $\mathrm{D} p$ brane are given by

$$
\begin{gathered}
f(t, z)=\sum_{\alpha=1}^{N} f_{(\alpha)}+\sum_{\alpha<\beta}^{N} f_{(\alpha)} f_{(\beta)} \sin ^{2}\left(\theta_{\alpha}-\theta_{\beta}\right), \\
f_{(\alpha)}(t, z)=\tilde{f}_{(\alpha)}(t)+\bar{f}_{(\alpha)}(z) \\
\tilde{f}_{(\alpha)}(t)=c_{(\alpha) 0} t+c_{(\alpha) 1} \\
\bar{f}_{(\alpha)}(z)=\bar{c}_{\alpha}+\frac{Q_{\alpha}}{\left|z^{a}-z_{(\alpha)}^{a}\right|^{5-p-d_{\mathrm{Z}}}}
\end{gathered}
$$

where $c_{(\alpha) 0}, c_{(\alpha) 1}, \bar{c}_{\alpha}$ are constants, and the rotation matrices $R_{(\alpha)}$ are defined by Eq. (25). $d_{\mathrm{Z}}(=0, \ldots, 6-p)$ denotes the number of smeared dimensions in $\mathrm{Z}$ space. In the case of $d_{\mathrm{Z}}=5-p$, the power function must be replaced by $\ln \left|z^{a}-z_{(\alpha)}^{a}\right|$. $Q_{\alpha}(\alpha=1, \ldots, N)$ are the charges (or masses) of $\mathrm{D} p$ branes and each $\mathrm{D} p$ brane is located at $z_{(\alpha)}^{a}$, respectively.

\section{PROPERTIES OF THE Dp-BRANE SYSTEM WITH ANGLES}

\section{A. Asymptotic structure}

The asymptotic structure of the dynamical $\mathrm{D} p$-brane solution is completely different from static ones. The static solution has an asymptotically flat geometry, but the present solution is time dependent. In fact, from Eq. (32) in the limit of $|z| \rightarrow \infty$, we find

$$
\begin{aligned}
d s^{2}= & -d \tau^{2}+\left(\frac{\tau}{\tau_{0}}\right)^{\frac{2(p-7)}{p+9}}\left[\gamma_{i j}(\mathrm{Y}) d y^{i} d y^{j}+\delta_{m n} d \zeta^{m} d \zeta^{n}\right] \\
& +\left(\frac{\tau}{\tau_{0}}\right)^{\frac{2(p+1)}{p+9}} u_{a b}(\mathrm{Z}) d z^{a} d z^{b}
\end{aligned}
$$

where we have defined the cosmic time $\tau$ by

$$
\tau=\tau_{0}\left(c_{(\alpha) 0} t+c_{(\alpha) 1}\right)^{\frac{16}{p+2}} .
$$

Hence our solution approaches asymptotically the anisotropic universe. The fastest expanding case is $\tau^{(p+1) /(p+9)}$ for $\mathrm{Z}$, and the part of $\mathrm{Y}$ spaces. So, if the static solution gives a $\mathrm{D} p$ brane, then we can regard the present solution as a $\mathrm{D} p$ brane in the expanding universe. The asymptotic behavior of the solution is sourced by the gravity and the time-dependent dilaton for $p \neq 3$ because the field strength vanishes at $|z| \rightarrow \infty$.

One important result with nontrivial angles $\left(\theta_{\alpha} \neq \theta_{\beta}\right)$ is that the nonlinear interaction term between branes appears as in Eq. (32a). From the solution (32), we find that the world volume of the time-dependent brane system in ten dimensions for the static observer is given by $\sqrt{-g} \propto h^{(p+1) / 8}$. Hence, if $c_{(\alpha) 0}<0$, it is contracting . Moreover, the transverse space to the $\mathrm{D} p$ brane always contracts as $h^{(p+1) / 16}$. For the case with $c_{(\alpha) 0}>0$, we find the opposite behavior.

\section{B. Spacetime singularity}

A curvature singularity appears at $h=0$ in the tendimensional spacetime. The regular region in tendimensional spacetime is obtained if and only if $h>0$. This regular spacetime region is bounded by curvature singularities.

If $d_{\mathrm{Z}} \leq 4-p$, although $h$ diverges on the branes, we find a regular spacetime at infinity. For $d_{\mathrm{Z}}=5-p$, since we have

$$
\bar{f}_{(\alpha)}(z)=Q_{k} \ln \left|z^{a}-z_{(\alpha)}^{a}\right|,
$$

the harmonic function $h$ diverges at infinity $\left(\left|z^{a}-z_{(\alpha)}^{a}\right| \rightarrow \infty\right)$ as well as near D $p$ branes $\left(\left|z^{a}-z_{(\alpha)}^{a}\right| \approx 0\right)$. Hence such a solution may not be physically relevant. For $d_{\mathrm{Z}}=6-p$, the functions $h$ are given by a sum of linear functions of $z$. Then this solution gives a regular behavior near branes, although the spacetime is not asymptotically flat.

As a result, the physically relevant solutions are classified by their behaviors into two classes: $d_{\mathrm{Z}} \leq 4-p$ and $d_{\mathrm{Z}}=6-p$. The collision of branes shows different behaviors as we see later.

\section{Compactified spacetime}

Since some dimensions are homogeneous, we can discuss the dynamics of a compactified spacetime. We compactify some dimensions of homogeneous spaces (Y space, $\zeta^{m}$ space, and $d_{\mathrm{Z}}$-dimensional smeared $\mathrm{Z}$ space). We assume that $\mathrm{Y}$ space, $\zeta^{m}$ space and $d_{c}\left(\leq d_{\mathrm{Z}}\right)$ dimensions in smeared $\mathrm{Z}$ space are compacitified. As a result, we find $(d+1)$-dimensional inhomogeneous compactified spacetime in the Einstein frame as

$$
d s_{d+1}^{2}=-h^{\frac{2-d}{d-1}} d t^{2}+h^{\frac{1}{d-1}} d z_{d}^{2},
$$

where $d=7-p-d_{c}$.

In the far region from the branes, since $h \approx c_{(\alpha) 0} t$ for $c_{(\alpha) 0}>0$, this spacetime expands as $\left|\tau-\tau_{0}\right|^{\frac{1}{d}}$, where $\tau$ is the cosmic time in the $(d+1)$-dimensional spacetime and $\tau_{0}$ is an integration constant, which is given by $\left(\tau-\tau_{0}\right) \propto \frac{d}{t^{2(d-1)}}$. However, since this is an inhomogeneous $(d+1)$ dimensional spacetime, it cannot describe our Universe. 
It does not describe a time-dependent black hole system either because of the present too simple brane configuration [8-10].

In order to discuss cosmology, we must find our three spaces in the homogeneous spaces ( $\mathrm{Y}$ space, $\zeta^{m}$ space, and a subset of $d_{\mathrm{Z}}$-dimensional smeared $\mathrm{Z}$ space) by fixing $z^{a}$ coordinates of unsmeared $\mathrm{Z}$ space. This means that our Universe is described by a test 3-brane located at some point $z^{a}=z_{(0)}^{a}$.
Let us consider the case of $N=2$. If we choose our three space in Y space, we find our four-dimensional spacetime in the Einstein frame as

$$
d s_{4}^{2}=h^{\alpha} \lambda^{-1 / 2}\left(-d t^{2}+\bar{\gamma}_{\mu \nu}\left(\mathrm{Y}^{\prime}\right) d y^{\mu} d y^{\nu}\right),
$$

where $\bar{\gamma}_{\mu \nu}\left(\mathrm{Y}^{\prime}\right)(\mu=1,2,3), \alpha$, and $\lambda$ are given by

$$
\bar{\gamma}_{\mu \nu}\left(\mathrm{Y}^{\prime}\right)=\left(\begin{array}{ccc}
1+f_{1}+f_{2} \cos ^{2} \theta & -f_{2} \sin \theta \cos \theta & 0 \\
-f_{2} \sin \theta \cos \theta & 1+f_{2} \sin ^{2} \theta & 0 \\
0 & 0 & 1+f_{1}+f_{2} \cos ^{2} \theta
\end{array}\right)
$$

$$
\begin{aligned}
\alpha & =\frac{1}{16}(p+1)\left(p+d_{\mathrm{Z}}-7\right)+\frac{1}{2}, \\
\lambda & =1+f_{1}+f_{2} \cos ^{2} \theta .
\end{aligned}
$$

The metric $\bar{\gamma}_{\mu \nu}\left(\mathrm{Y}^{\prime}\right)$ describes anisotropic expansion of the three-dimensional universe even if $\bar{\gamma}_{\mu \nu}\left(\mathrm{Y}^{\prime}\right)$ is diagonalized. Upon setting $f_{1} \approx c_{0} t\left(c_{0}>0\right)$ and $\partial_{t} f_{2}=0$, the Universe expands as $\left|\tau-\tau_{0}\right|^{\frac{\beta+1}{\beta+2}}$, for the fastest expanding case. Here the cosmic time $\tau$ and the constant $\beta$ are given by

$$
\tau=\frac{2}{c_{0}(\beta+2)}\left(c_{0} t\right)^{\frac{\beta+2}{2}}, \quad \beta=\alpha-\frac{1}{2} .
$$

In what follows, using the solutions obtained in this paper, we discuss the collision of D3 branes with different angles in ten-dimensional spacetime.

\section{COLLISION OF THE DYNAMICAL D3 BRANE}

We now discuss collision of two D3 branes with different angles. The behavior of the harmonic function $\bar{f}_{(\alpha)}(z)$ is classified into two classes depending on the number of smeared dimensions of the D3 brane, that is, $d_{\mathrm{Z}} \leq 1$ and $d_{\mathrm{Z}}=3$, which we discuss below separately. For $d_{\mathrm{Z}}=2$, the harmonic function $\bar{f}_{(\alpha)}(z)$ diverges both at infinity and near D3 branes.

\section{A. $d_{\mathrm{Z}} \leq 1$}

The harmonic function $h$ becomes dominant in the limit of $z^{a} \rightarrow z_{(\alpha)}^{a}(\alpha=1$ and 2$)$. Hence, we recover a static spacetime of the $\mathrm{D} p$-brane system near branes. On the other hand, the function $h$ depends only on time $t$ in the limit of $\left|z^{a}\right| \rightarrow \infty$. As a result, in the far region from branes, the homogeneous spacetime is found.

In order to analyze the brane collision, we consider a concrete example as follows: Two D3 branes are located at $z_{(1)}^{a}=\left(z_{(1)}, 0, \ldots, 0\right)$ and $z_{(2)}^{a}=\left(z_{(2)}, 0, \ldots, 0\right)$. We assume that $\tilde{f}_{(1)}$ is time dependent, and we discuss the time evolution separately with respect to the signature of a constant $c_{(1) 0}$, because the behavior of spacetime strongly depends on it. Since the spacetime is singular at $h(t, z)=0$, the regular spacetime is obtained inside the spacetime region restricted by

$$
h(t, z)=1+f(t, z)>0,
$$

where the function $f(t, z)$ is given by

$$
\begin{aligned}
f(t, z)= & c_{(1) 0} t+c_{(1) 1}+c_{(2) 1}+\frac{Q_{1}}{\left|z^{a}-z_{(1)}^{a}\right|^{2-d_{\mathrm{Z}}}} \\
& +\frac{Q_{2}}{\left|z^{a}-z_{(2)}^{a}\right|^{2-d_{\mathrm{Z}}}} .
\end{aligned}
$$

We have set $\bar{c}_{1}=\bar{c}_{2}=0$ without loss of generality. Since the spacetime cannot be extended beyond this region, the regular spacetime with two D3 branes ends on these singular hypersurfaces. The solution with $c_{(1) 0}>0$ is the time reversal one of $c_{(1) 0}<0$, because the time dependence appears only in the form of $c_{(1) 0} t$. In the following, we consider the case with $c_{(1) 0}<0$.

For $c_{(1) 0}<0$ and the appropriate choice of the constant $c$, if $Q_{1}, Q_{2}>0$, the ten-dimensional spacetime is nonsingular at the initial time $(t=0)$ because the function $h$ is positive everywhere. In the limit of $t \rightarrow-\infty$, the tendimensional spacetime becomes asymptotically a timedependent uniform background except for the cylindrical forms of infinite throats near branes $\left(\left|z^{a}-z_{(\alpha)}^{a}\right| \approx 0\right)$.

As time evolves $(t>0)$, the singularity appears from a far region $\left(\left|z-z_{(\alpha)}\right| \rightarrow \infty\right)$ and the singular hypersurface erodes the region with the large values of $\left|z-z_{(\alpha)}\right|$. As a result, only the region of near D3 branes remains regular. A singular hypersurface eventually surrounds each D3 brane individually and then the regular regions near D3 branes split into two isolated throats. 


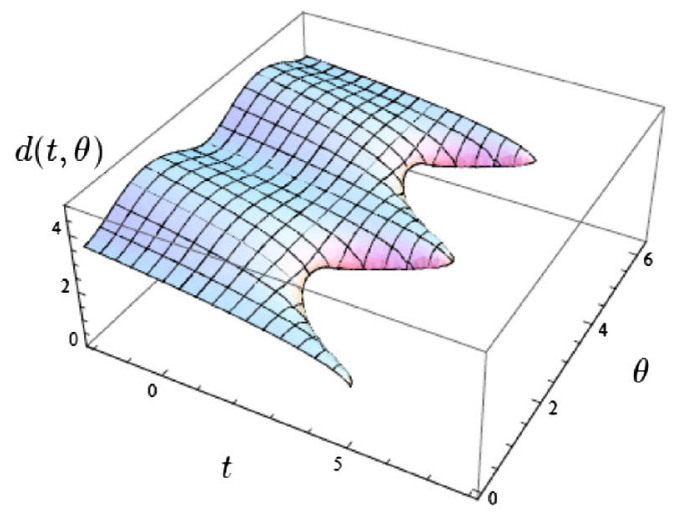

(a)

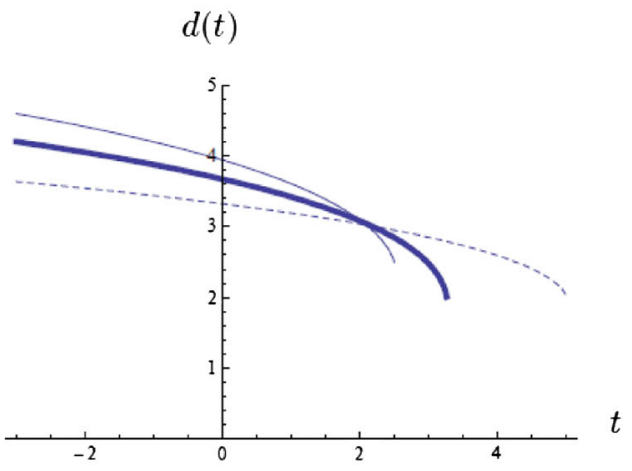

(b)

FIG. 1. (a) For the case of $Q_{1}=Q_{2}$ in the dynamical D3 branes, the proper distance between two dynamical D3 branes given in (42) is depicted. We fix $d_{\mathrm{Z}}=1, c_{(1) 0}=-1, Q_{1}=Q_{2}=1, z_{(1)}=-1, z_{(2)}=1$, and $c_{(1) 1}=c_{(2) 1}=1$ for the angle $0 \leq \theta \leq 2 \pi$. A singularity appears between two D3 branes and the spacetime splits into two isolated brane throats before they collide. (b) We also show the proper distance $d(t)$ between two dynamical D3 branes for $\theta=0$ (dashed curve), $\theta=\pi / 4$ (bold curve) and $\theta=\pi / 2$ (solid curve) from the bottom in the case of $d_{\mathrm{Z}}=1, Q_{1}=Q_{2}=1, z_{(1)}=-1, z_{(2)}=1$, and $c_{(1) 0}=-1$ in the dynamical D3-brane system. Although the proper distance decreases as $t$ increases, the distance is still finite when a singularity appears.

In Fig. 1 , for the case of $d_{\mathrm{Z}}=1$, we show the proper distance $d(t, \theta)$ between two branes at $z_{(1)}^{a}$ and $z_{(2)}^{a}$, which is defined by

$$
\begin{aligned}
d(t, \theta)= & \int_{z_{(1)}}^{z_{(2)}} d z\left[c_{(1) 0} t+c_{(1) 1}+c_{(2) 1}+1+\frac{Q_{1}}{\left|z-z_{(1)}\right|^{2-d_{\mathrm{Z}}}}\right. \\
& +\frac{Q_{2}}{\left|z-z_{(2)}\right|^{2-d_{\mathrm{Z}}}}+\left(c_{(1) 0} t+c_{(1) 1}+\frac{Q_{1}}{\left|z-z_{(1)}\right|^{2-d_{\mathrm{Z}}}}\right) \\
& \left.\times\left(c_{(2) 1}+\frac{Q_{2}}{\left|z-z_{(2)}\right|^{2-d_{\mathrm{Z}}}}\right) \sin ^{2} \theta\right]^{1 / 4}
\end{aligned}
$$

where we have defined $z \equiv z^{1}$, and $d_{\mathrm{Z}}$ denotes the number of smeared dimension. This is a monotonically decreasing function of $t$ if $c_{(1) 0}<0$. In Fig. 1, we choose $c_{(1) 0}=-1$, $Q_{1}=Q_{2}=1$ and $z_{(1)}=-z_{(2)}=-1$.

Figure 1 shows that a singularity appears earlier as the angle $\theta$ increases from 0 to $\pi / 2$, but the singularity always appears before the distance $d$ vanishes. Then, a singularity between two D3 branes forms before they collide into each other.

There is no qualitative difference when we have the different magnitudes of charges. Two branes approach very slowly, but a singularity eventually appears at a finite distance and the spacetime splits into two isolated D3-brane throats.

We should also mention the case with different signs of charges (a system of the D3 brane and anti-D3 brane). If $Q_{1}$ and $Q_{2}$ have different signs, we find that there always exists a singularity between two branes. Hence we cannot even set up such a situation from the beginning. It is, however, not the case for $d_{\mathrm{Z}}=3$ (see the next subsection).

Hence, we cannot discuss a D3-brane collision in the case of $d_{\mathrm{Z}} \leq 1$.

\section{B. $d_{\mathrm{Z}}=3$}

Next we consider the case $d_{\mathrm{Z}}=3$. We set two D3 branes with a brane charge $Q_{1}$ at $z=z_{(1)}$ and the other $Q_{2}$ at $z=z_{(2)}$. The solution for $\bar{f}_{(\alpha)}(z)$ is obtained explicitly as

$$
\bar{f}_{(\alpha)}(z)=Q_{k}\left|z-z_{(\alpha)}\right|,
$$

where $Q_{k}$ and $z_{(\alpha)}$ are constant parameters. We set $\bar{c}_{\alpha}=0$ without loss of generality.

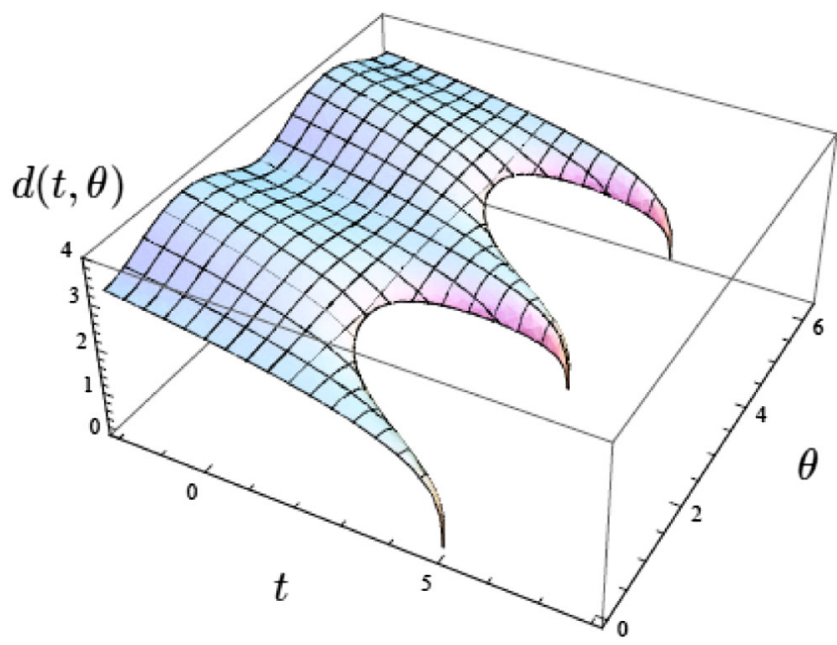

FIG. 2. For the case of $Q_{1}=Q_{2}$ in the dynamical D3 branes, the proper distance between two dynamical D3 branes given in (42) is depicted. We fix $d_{\mathrm{Z}}=3, c_{(1) 0}=-1, Q_{1}=Q_{2}=1$, $z_{(1)}=-1, z_{(2)}=1$, and $c_{(1) 1}=c_{(2) 1}=1$ for $0 \leq \theta \leq 2 \pi$. The distance decreases, and then two D3 branes collide into each other. The proper distance rapidly vanishes near two branes colliding for the case of $\theta=0, \theta=\pi, \theta=2 \pi$. 


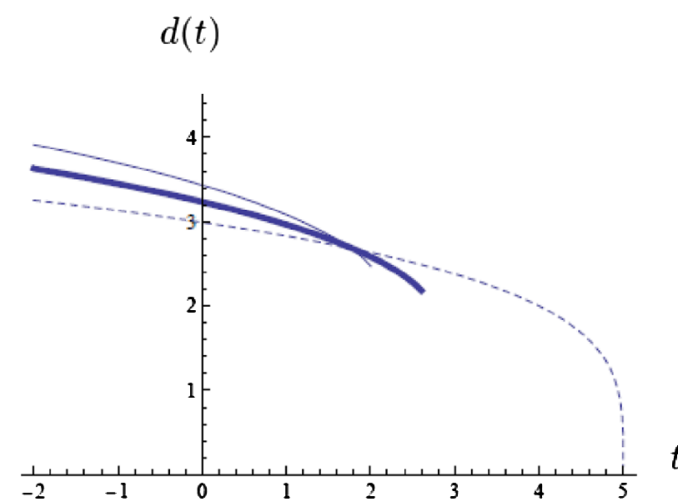

(a) $d(\theta)$

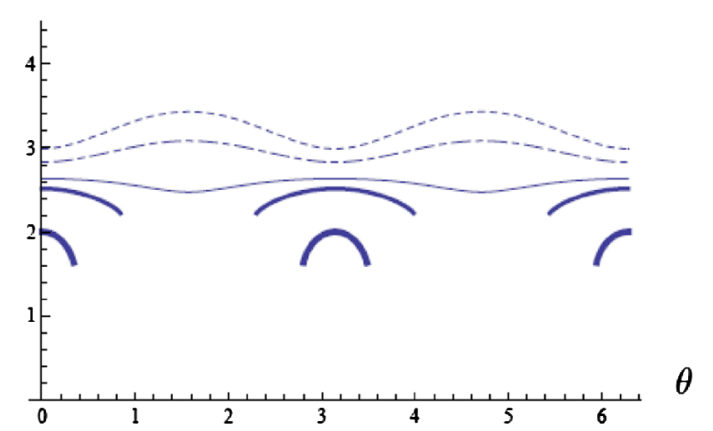

(b)

FIG. 3. (a) We show the proper distance between two dynamical D3 branes for $\theta=0$ (dashed curve), $\theta=\pi / 4$ (bold curve) and $\theta=\pi / 2$ (solid curve) from the bottom in the case of $d_{\mathrm{Z}}=3, Q_{1}=Q_{2}=1, z_{(1)}=-1, z_{(2)}=1$, and $c_{(1) 0}=-1$ in the dynamical D3brane system. If we set $\theta=0$, it causes the complete collision at $t=5$ simultaneously. For the case of $\theta \neq n \pi$ ( $n=$ integer), it is still finite when a singularity appears. (b) We also show the snapshots at $t=0$ (dashed line), $t=1$ (dashed-dotted line), $t=2$ (solid line), $t=2.5$ (bold line), and $t=4$ (thick bold line). The proper distance $d(t, \theta)$ vanishes at $t=5, \theta=n \pi(n=$ integer).

The proper distance between two D3 branes is given by

$$
\begin{aligned}
d(t, \theta)= & \int_{z_{(1)}}^{z_{(2)}} d z\left[c_{(1) 0} t+c_{(1) 1}+c_{(2) 1}+1+Q_{1}\left|z-z_{(1)}\right|\right. \\
& +Q_{2}\left|z-z_{(2)}\right|+\left(c_{(1) 0} t+c_{(1) 1}+Q_{1}\left|z-z_{(1)}\right|\right) \\
& \left.\times\left(c_{(2) 1}+Q_{2}\left|z-z_{(2)}\right|\right) \sin ^{2} \theta\right]^{1 / 4} .
\end{aligned}
$$

The proper distance is a monotonically decreasing function of $t$ if we set again $c_{(1) 0}<0$. We illustrate $d(t, \theta)$ for the case of the D3-brane system in Fig. 2. We also depict the proper distance $d(t)$ for given angles $\theta=0, \pi / 4$ and $\pi / 2$, and the angular dependence of the proper distance $d(\theta)$ at some particular times $t=0,1,2$,

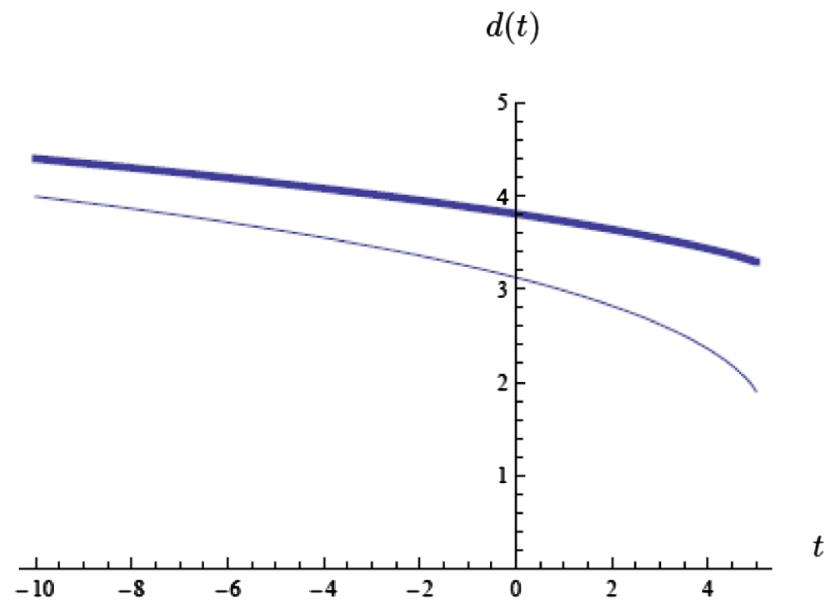

FIG. 4. We depict the proper distance between two dynamical D3 branes for the cases of $10 Q_{1}=Q_{2}=10$ (thick bold line) and $2 Q_{1}=Q_{2}=2$ (solid curve). We choose $\theta=0, d_{\mathrm{Z}}=3$, $z_{(1)}=-1, z_{(2)}=1$, and $c_{(1) 0}=-1$. All proper distances are still finite when a singularity appears.
2.5, 4 and 5 in Fig. 3. We set $c_{(1) 0}=-1, c_{(1) 1}=c_{(2) 1}=1$, $z_{(1)}=-1, z_{(2)}=1$, and $Q_{1}=Q_{2}=1$.

The angle dependence is similar to the case of $d_{\mathrm{Z}} \leq 1$. The proper distance $d$ between two branes never vanishes. For the case of the equal charges $\left(Q_{1}=Q_{2}\right)$, however, there is one exceptional case, which is the two D3-brane system with the trivial angle $(\theta=0$ or $\pi)$. In this case, as we can see in Figs. 2 and 3, $d$ vanishes just when a singularity appears. Two D3 branes collide completely and a singularity appears at the same time. Hence we conclude that two

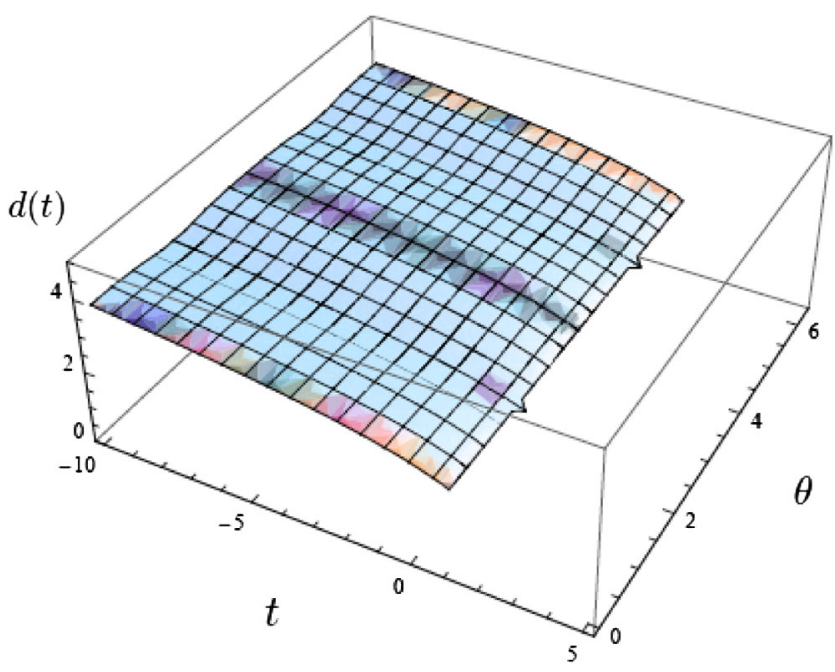

FIG. 5. For the case of $Q_{1}=-Q_{2}$ in the dynamical D3 branes, the proper distance between two dynamical D3 branes given in (42) is depicted. We fix $d_{\mathrm{Z}}=3, c_{(1) 0}=-1, Q_{1}=-Q_{2}=1$, $z_{(1)}=-1, z_{(2)}=1$, and $c_{(1) 1}=c_{(2) 1}=1$ for the $0 \leq \theta \leq 2 \pi$. In this case, a singularity appears at $t<0$ when the distance is still finite. Then, the solution does not describe the collision of two D3 branes. 


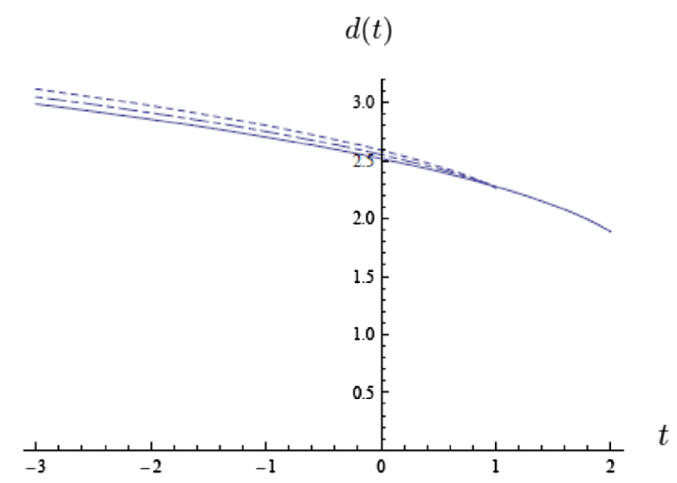

(a) $d(\theta)$

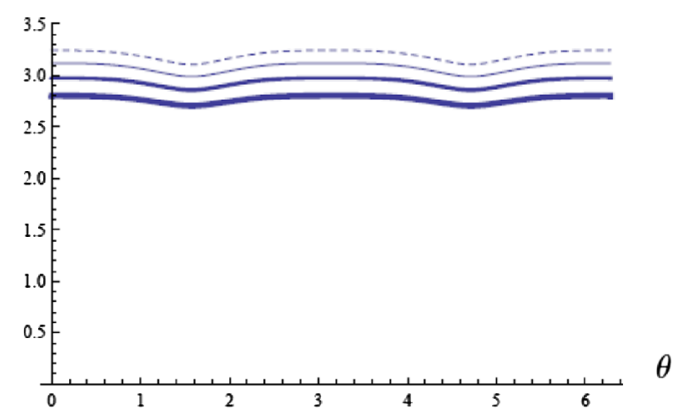

(b)

FIG. 6. (a) We show the proper distance at $\theta=0$ (dotted line), $0.35 \pi$ (dashed-dotted line), and $0.5 \pi$ (solid line) in the case of the dynamical D3-brane system. We set $d_{\mathrm{Z}}=3, Q_{1}=-Q_{2}=1, z_{(1)}=-1, z_{(2)}=1$, and $c_{(1) 0}=-1$. Although the proper distance decreases as $t$ increases, the distance is still finite when a singularity appears at $t<0$. (b) We also depict the snapshots at $t=-4$ (dashed line), $t=-3$ (solid line), $t=-2$ (bold line), and $t=-1$ (thick bold line). Although the proper distance decreases as $t$ increases, the distance is still finite when a singularity appears at $t<0$.

branes will never collide into each other except in the case with the trivial angles.

How about the different values of charges? We then show the case with different magnitudes of charges in Fig. 4. In this figure, we have just shown the case with $\theta=0$, which may give the closest distance when a singularity appears. This figure shows that any two-brane system with the different magnitudes of charges never collides when a singularity appears.

We also show the proper distance between two branes for the case with different signs of charges such that $Q_{1}=1$ and $Q_{2}=-1$ in Figs. 5 and 6 . In this case, it describes the system of the D3 brane and the anti-D3 brane with the same masses of $M_{1}=M_{2}=1$.

Although two branes approach each other, a singularity always appears before two branes collide. It is different

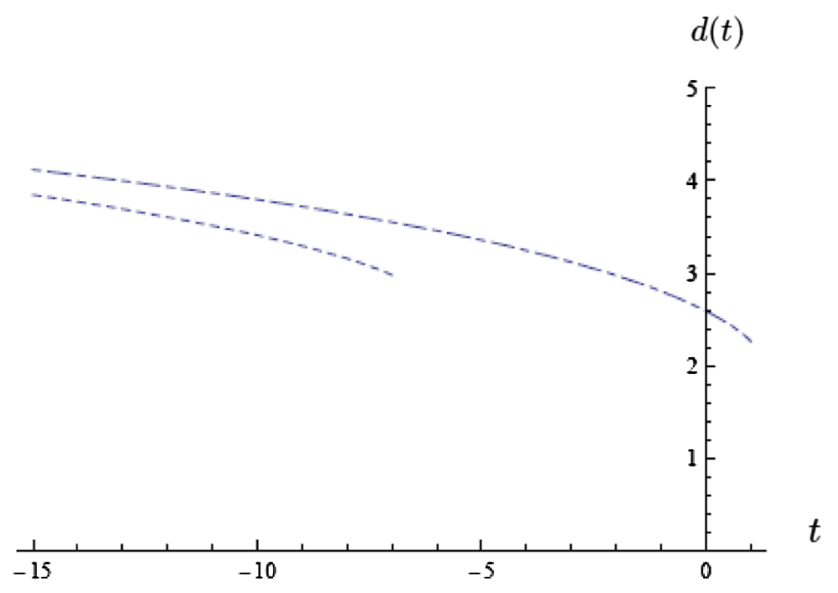

FIG. 7. We depict the proper distance between two dynamical branes for the cases of $Q_{1}=-Q_{2}=1$ (dashed-dotted line) and $5 Q_{1}=-Q_{2}=5$ (dashed curve). We choose $\theta=0, d_{\mathrm{Z}}=3$, $z_{(1)}=-1, z_{(2)}=1$, and $c_{(1) 0}=-1$. All proper distances are still finite when a singularity appears. from the case with the same charges $\left(Q_{1}=Q_{2}\right)$, in which two branes can collide when $\theta=0$ or $\pi$. The proper distance $d(t, \theta)$ never vanishes for the present case. This result does not change even if the magnitudes of two charges are different, as we show in Fig. 7.

We then conclude that for the case of $d_{\mathrm{Z}}=3$, only the two-brane system with the same charges as well as with the trivial angle $(\theta=0$ or $\pi)$ gives a complete brane collision.

Since the collision of the $\mathrm{D} p$ brane without angles strongly depends on the form of harmonic functions, there does not seem to be any novel feature due to the angles of the branes. However, for the dynamical $\mathrm{D} p$-brane background with angles, the angle couples to time coordinates in the ten-dimensional metric (30). Then, upon setting $c_{(\alpha) 0}<0$, the function $h(t, z)$ first vanishes at $\sin \theta= \pm 1$, which corresponds to the curvature singularity in the background. As the time evolves, the subsequent vanishing of $h(t, z)$ occurs at $\sin \theta \neq 0$, before the proper distance between the $\mathrm{D}$ branes vanishes, i.e., a singularity forms before the collision of two branes.

\section{DISCUSSION}

In the present paper, we have discussed the dynamical $\mathrm{D} p$-brane solution which describes several $\mathrm{D} p$ branes oriented at angles with respect to one another. Since the corresponding background field configurations remain largely unexplored, we have presented one such class of solutions in the time-dependent $\mathrm{D} p$-brane background. The dynamical solution which we have obtained in this paper describes any number of $\mathrm{D} p$ branes whose relative orientations are given by certain SU(2) rotations. These are functions of time that become static near $\mathrm{D}$ branes with the simplest possible dependence on the warp factor. In the far region from the angled $\mathrm{D} p$ brane in the ten-dimensional background, the solutions give a purely contracting or expanding uniform universe. 
We have also discussed the dynamics of angled D3-brane models with applications to collision of branes. The two D3 branes approach at an angle $\theta$ in the direction of the spatial part of world volume coordinates $y^{i}$. We have studied the dynamics of D3 branes which have been smeared along the transverse space to D3 branes. If two D3-brane charges are different from each other $\left(Q_{1}=-Q_{2}\right)$, the distance between two D3 branes is still finite when a singularity appears. Thus, we cannot describe the collision of two D3 branes in terms of the solution. For $d_{\mathrm{Z}} \leq 1$, a singularity again appears before D3 branes collide. Then, the topology of the spacetime eventually changes so that branes are separated by singular hypersurfaces surrounding each D3 brane. This behavior also appears if $Q_{1}=Q_{2}$ and $d_{\mathrm{Z}}=3$. A singularity forms at $\theta \neq 0, \pi$, when the distance is still finite. On the other hand, it has been shown that there exists a complete collision where the orientation of configurations between two smeared D3 branes with $Q_{1}=Q_{2}$ is either 0 or $\pi$. This result may be related to supersymmetry, which may be broken for nonparallel branes.

Although the examples presented in the present paper cannot provide a realistic cosmological model, the solution may be utilized to construct a cosmological solution just by introduction of a test brane universe in higher dimensions. We may also construct a new type of time-dependent black hole solution with nontrivial angles by setting up a more complicated brane configuration. Those subjects are left for future works.

\section{ACKNOWLEDGMENTS}

This work is supported by Grants-in-Aid from the Scientific Research Fund of the Japan Society for the Promotion of Science (Grants No. 25400276, No. 16K05362 and No. 16K05364).

\section{APPENDIX: DYNAMICAL SOLUTIONS OF D3- AND D4-BRANE SYSTEMS WITH ANGLES}

In this appendix, we show how to find the solution of a dynamical $\mathrm{D} p$-brane system $(p>2)$ by use of a T duality. We present the explicit procedure for the cases of D3- and D4-brane systems. In order to find the configurations of a higher-dimensional $\mathrm{D} p$-brane system with angles $(p \geq 5)$, we can repeat the same procedure.

\section{D3 brane with angle}

The D3-brane system with angle is obtained by a Tduality transformation from the solutions presented in Sec. II A. Let us consider the D2-brane solutions (24). To perform a $\mathrm{T}$ duality along the $z^{5}$ direction, we have to smear and delocalize the solution in this direction. Hence $h$ does not depend on $z^{5}$.

For the string frame, the ten-dimensional T-duality map from the type-IIA theory to type-IIB theory is given by $[30,38]$

$$
\begin{aligned}
\bar{g}_{\zeta \zeta}^{(\mathrm{B})} & =\frac{1}{\bar{g}_{\zeta \zeta}^{(\mathrm{A})}}, \quad \bar{g}_{M N}^{(\mathrm{B})}=\bar{g}_{M N}^{(\mathrm{A})}-\frac{\bar{g}_{\zeta M}^{(\mathrm{A})} \bar{g}_{\zeta N}^{(\mathrm{A})}-B_{\zeta M}^{(\mathrm{A})} B_{\zeta N}^{(\mathrm{A})}}{\bar{g}_{\zeta \zeta}^{(\mathrm{A})}}, \\
\bar{g}_{\zeta M}^{(\mathrm{B})} & =-\frac{B_{\zeta M}^{(\mathrm{A})}}{\bar{g}_{\zeta \zeta}^{(\mathrm{A})}}, \\
\mathrm{e}^{2 \phi_{(\mathrm{B})}} & =\frac{\mathrm{e}^{2 \phi_{(\mathrm{A})}}}{\bar{g}_{\zeta \zeta}^{(\mathrm{A})}}, \quad B_{M N}^{(\mathrm{B})}=B_{M N}^{(\mathrm{A})}+2 \frac{\bar{g}_{\zeta[M}^{(\mathrm{A})} B_{N] \zeta}^{(\mathrm{A})}}{\bar{g}_{\zeta \zeta}^{(\mathrm{A})}}, \quad B_{\zeta M}^{(\mathrm{B})}=-\frac{\bar{g}_{\zeta M}^{(\mathrm{A})}}{\bar{g}_{\zeta \zeta}^{(\mathrm{A})},} \\
C_{M N} & =C_{M N \zeta}-2 C_{[M} B_{N] \zeta}^{(\mathrm{A})}+2 \frac{\bar{g}_{\zeta[M}^{(\mathrm{A})} B_{N] \zeta}^{(\mathrm{A})} C_{z}}{\bar{g}_{\zeta \zeta}^{(\mathrm{A})}}, \\
C_{\zeta M} & =C_{M}-\frac{C_{\zeta}^{(\mathrm{A})} \bar{g}_{\zeta M}^{(\mathrm{A})}}{\bar{g}_{\zeta \zeta}^{(\mathrm{A})}}, \\
C_{M N P \zeta} & =C_{M N P}-\frac{3}{2}\left(C_{[M} B_{N P]}^{(\mathrm{A})}-\frac{\bar{g}_{\zeta[M}^{(\mathrm{A})} B_{N P]}^{(\mathrm{A})} C_{\zeta}}{\bar{g}_{\zeta \zeta}^{(\mathrm{A})}}+\frac{\bar{g}_{\zeta[M}^{(\mathrm{A})} C_{N P] \zeta}}{\left.\bar{g}_{\zeta \zeta}^{(\mathrm{A})}\right),}\right. \\
C_{(0)} & =-C_{\zeta},
\end{aligned}
$$

where $\bar{g}_{M N}$ denotes the ten-dimensional metric in the string frame performing a Weyl rescaling of the metric in Einstein frame, $\bar{g}_{M N}=\mathrm{e}^{\phi / 2} g_{M N}, \zeta=z^{5}$ is the coordinate to which the T dualization is applied, and $M, N, P$ denote the other coordinates: $0, y^{i}(i=1, \ldots, 4)$, and $z^{a}(a=1, \ldots, 4)$. Via the T-duality map (A1), we obtain the Einstein frame metric of the dynamical $N$ D3-brane system in ten-dimensional background as [30,34]

$$
\begin{aligned}
d s^{2} & =g_{M N} d x^{M} d x^{N} \\
& =h^{-1 / 2}\left[-d t^{2}+\gamma_{i j} d y^{i} d y^{j}+d \zeta^{2}+h u_{a b}(z) d z^{a} d z^{b}\right],
\end{aligned}
$$

$$
\begin{gathered}
\gamma_{i j} d y^{i} d y^{j}=\delta_{i j} d y^{i} d y^{j}+\sum_{\alpha=1}^{N} f_{(\alpha)}\left[\left\{\left(R_{(\alpha)}\right)^{1}{ }_{i} d y^{i}\right\}^{2}\right. \\
\left.+\left\{\left(R_{(\alpha)}\right)^{3}{ }_{j} d y^{j}\right\}^{2}\right] \\
h(t, z)=1+f(t, z)
\end{gathered}
$$

where the function $f$ and the rotation matrix $R_{(\alpha)}$ associated with the $\alpha$ th D3 brane are given by (25).

This describes the $N$ D3-brane system such that each brane first lying in the $\left(y^{2}, y^{4}, \zeta\right)$ space rotates by an angle $\theta_{\alpha}$ $(\alpha=1, \ldots, N)$ in the $y^{1}-y^{2}$ and $y^{3}-y^{4}$ planes as $(\xi, \eta) \rightarrow$ $\left(\mathrm{e}^{i \theta_{\alpha}} \xi, \mathrm{e}^{-i \theta_{\alpha}} \eta\right)$ where $\xi=y^{1}+i y^{2}$ and $\eta=y^{3}+i y^{4}$, the rotation of which belongs to the $\mathrm{SU}(2)$ group.

Since we apply the T-duality map from the type-IIA to the type-IIB theory along $\zeta=z^{5}$, the smeared-out solution yields 


$$
\begin{aligned}
F_{(5)}= & (1 \pm *) d\left(h^{-1}\right) d t \wedge d y^{5} \\
& \wedge\left\{\sum_{\alpha=1}^{N} f_{(\alpha)}\left(R_{(\alpha)}\right)_{i}^{2} d y^{i} \wedge\left(R_{(\alpha)}\right)_{j}^{4} d y^{j}\right. \\
& \left.-\sum_{a<b}^{N} f_{(\alpha)} f_{(\beta)} \sin ^{2}\left(\theta_{\alpha}-\theta_{\beta}\right)\left(d y^{1} \wedge d y^{3}-d y^{2} \wedge d y^{4}\right)\right\},
\end{aligned}
$$

$$
\mathrm{e}^{2 \phi}=\mathrm{e}^{2 \phi_{0}}
$$

where $\phi_{0}$ is constant and $*$ denotes the Hodge dual operator in the ten-dimensional background. Using the above results, the field equations give

$$
\begin{gathered}
R_{a b}(\mathrm{Z})=0 \\
f_{(\alpha)}(t, z)=\tilde{f}_{(\alpha)}(t)+\bar{f}_{(\alpha)}(z), \\
\partial_{t}^{2} \tilde{f}_{(\alpha)}=0, \quad \partial_{t} \tilde{f}_{(\alpha)} \partial_{t} \tilde{f}_{(\beta)}=0(\alpha \neq \beta), \quad \Delta_{\mathrm{Z}} \bar{f}_{(\alpha)}=0 .
\end{gathered}
$$

This gives the exact solution for the $N$ D3-brane system with different angles for giving Ricci flat $\mathrm{Z}$ space. As the D2-brane system, only one brane can be time dependent,

$$
\tilde{f}_{(\alpha)}=c_{(\alpha) 0} t+c_{(\alpha) 1},
$$

where $c_{(\alpha) 0}, c_{(\alpha) 1}$ are constant, and the other $\tilde{f}_{(\beta)}=c_{(\beta) 1}$ $(\beta \neq \alpha)$ are constant.

For the metric $u_{a b}=\delta_{a b}$, where $\delta_{a b}$ are the fourdimensional flat Euclidean metric, we find the exact solution

$$
\bar{f}_{(\alpha)}(z)=\bar{c}_{\alpha}+\frac{Q_{\alpha}}{\left|z^{a}-z_{(\alpha)}^{a}\right|^{2-d_{\mathrm{Z}}}},
$$

where $\bar{c}_{\alpha}, Q_{\alpha}$ and $z_{(\alpha)}^{a}$ are constant parameters and $d_{\mathrm{Z}}(=0, \ldots, 3)$ is the smeared dimensions in the $\mathrm{Z}$ space. In the case of $d_{\mathrm{Z}}=2$, the power function must be replaced by $\ln \left|z^{a}-z_{(\alpha)}^{a}\right| . Q_{\alpha}$ denotes a charge (or mass) of the D3 brane and $z^{a}=z_{(\alpha)}^{a}$ is the position of the D3 brane.

\section{D4 brane with angle}

Next we give the dynamical solution of the $N$ D4-brane system after applying a T-duality in the $z^{4}$ direction of the ten-dimensional spacetime (A2). The T-duality relations from type-IIB to type-IIA theory are given by $[35,38-40]$

$$
\begin{aligned}
\bar{g}_{\zeta^{\prime} \zeta^{\prime}}^{(\mathrm{A})}= & \frac{1}{\bar{g}_{\zeta^{\prime} \zeta^{\prime}}^{(\mathrm{B})},} \quad \bar{g}_{M N}^{(\mathrm{A})}=\bar{g}_{M N}^{(\mathrm{B})}-\frac{\bar{g}_{\zeta^{\prime} M}^{(\mathrm{B})} \bar{g}_{\zeta^{\prime} N}^{(\mathrm{B})}-B_{\zeta^{\prime} M}^{(\mathrm{B})} B_{\zeta^{\prime} N}^{(\mathrm{B})}}{\bar{g}_{\zeta^{\prime} \zeta^{\prime}}^{(\mathrm{B})}}, \\
\bar{g}_{\zeta^{\prime} M}^{(\mathrm{A})}= & -\frac{B_{\zeta^{\prime} M}^{(\mathrm{B})}}{\bar{g}_{\zeta^{\prime} \zeta^{\prime}}^{(\mathrm{B})}}, \\
\mathrm{e}^{2 \phi(\mathrm{A})}= & \frac{\mathrm{e}^{2 \phi(\mathrm{B})}}{\bar{g}_{\zeta^{\prime} \zeta^{\prime}}^{(\mathrm{B})}}, \quad C_{M}=C_{\zeta^{\prime} M}+\chi B_{\zeta^{\prime} M}^{(\mathrm{B})}, \quad C_{\zeta}^{\prime}=-\chi, \\
B_{M N}^{(\mathrm{A})}= & B_{M N}^{(\mathrm{B})}+2 \frac{B_{\zeta^{\prime}[M}^{(\mathrm{B})} \bar{g}_{N] \zeta^{\prime}}^{(\mathrm{B})}}{\bar{g}_{\zeta^{\prime} \zeta^{\prime}}^{(\mathrm{B})}}, \quad B_{\zeta^{\prime} M}^{(\mathrm{A})}=-\frac{\bar{g}_{\zeta^{\prime} M}^{(\mathrm{B})}}{\bar{g}_{\zeta^{\prime} \zeta^{\prime}}^{(\mathrm{B})}}, \\
C_{\zeta^{\prime} M N}= & C_{M N}+2 \frac{C_{\zeta^{\prime}[M} \bar{g}_{N] \zeta^{\prime}}^{(\mathrm{B})}}{\bar{g}_{\zeta^{\prime} \zeta^{\prime}}^{(\mathrm{B})}}, \\
C_{M N P}= & C_{M N P \zeta^{\prime}}+\frac{3}{2}\left(C_{\zeta^{\prime}[M} B_{N P]}^{(\mathrm{B})}-B_{\zeta^{\prime}[M}^{(\mathrm{B})} C_{N P]}\right. \\
& \left.-4 \frac{B_{\zeta^{\prime}[M}^{(\mathrm{B})} C_{\left|\zeta^{\prime}\right| N} \bar{g}_{P] \zeta^{\prime}}^{(\mathrm{B})}}{\bar{g}_{\zeta^{\prime} \zeta^{\prime}}^{(\mathrm{B})}}\right),
\end{aligned}
$$

where $\zeta^{\prime}=z^{4}$ is the coordinate to which the $\mathrm{T}$ duality is performed. The indices $M, N$ and $P$ denote the other coordinates: $0, y^{i}(i=1, \ldots, 2), \zeta$ and $z^{a}(a=1,2,3)$. We delocalize the D3-brane solution in the transverse coordinate $\zeta^{\prime}=z^{4}$. With the relations in (A7), applying T-duality along this direction to produce a system of an $N$ D4-brane system with different angles, the type-IIA metric in the Einstein frame is given by

$$
\begin{aligned}
d s^{2}= & g_{M N} d x^{M} d x^{N} \\
= & h^{-3 / 8}\left[-d t^{2}+\gamma_{i j} d y^{i} d y^{j}+d \zeta^{2}+d \zeta^{12}\right. \\
& \left.+h u_{a b}(z) d z^{a} d z^{b}\right],
\end{aligned}
$$

$\gamma_{i j} d y^{i} d y^{j}=\delta_{i j} d y^{i} d y^{j}$

$$
+\sum_{\alpha=1}^{N} f_{(\alpha)}\left[\left\{\left(R_{(\alpha)}\right)^{1}{ }_{i} d y^{i}\right\}^{2}+\left\{\left(R_{(\alpha)}\right)_{j}^{3} d y^{j}\right\}^{2}\right]
$$

$$
h(t, z)=1+f(t, z),
$$

where the function $f$ and the rotation matrix $R_{(\alpha)}$ associated with the $\alpha$ th D4 brane is defined as (25). The other fields are a straightforward generalization of the case of a static D4-brane system with certain angles in the type-IIA low energy effective string theory [30,34],

$$
F_{(4)}=d y^{i} \wedge d y^{j} \epsilon_{i j k} \partial_{k}\left[\sum_{\alpha=1}^{N} f_{(\alpha)}\left(R_{(\alpha)}\right)_{\ell}^{2} d y^{\ell} \wedge\left(R_{(\alpha)}\right)_{m}^{4} d y^{m}\right],
$$




$$
\mathrm{e}^{2 \phi}=h^{-1 / 2}
$$

Then, the field equations reduce to

$$
\begin{gathered}
R_{a b}(\mathrm{Z})=0, \\
f_{(\alpha)}(t, z)=\tilde{f}_{(\alpha)}(t)+\bar{f}_{(\alpha)}(z), \\
\partial_{t}^{2} \tilde{f}_{(\alpha)}=0, \quad \partial_{t} \tilde{f}_{(\alpha)} \partial_{t} \tilde{f}_{(\beta)}=0, \quad(\alpha \neq \beta) \quad \Delta_{\mathrm{Z}} \bar{f}_{(\alpha)}=0 .
\end{gathered}
$$

(A11c)

This gives the exact solution for the $N$ D4-brane system with different angles for giving Ricci flat $\mathrm{Z}$ space. As for the other multibrane systems, only one brane can be time dependent,

$$
\tilde{f}_{(\alpha)}=c_{(\alpha) 0} t+c_{(\alpha) 1},
$$

where $c_{(\alpha) 0}, c_{(\alpha) 1}$ are constant, and the other $\tilde{f}_{(\beta)}=c_{(\beta) 1}$ $(\beta \neq \alpha)$ are constant.

For the metric $u_{a b}=\delta_{a b}$, where $\delta_{a b}$ is the threedimensional flat Euclidean metric, the harmonic functions $\bar{f}_{\alpha}$ are given by

$$
\bar{f}_{(\alpha)}(z)=\bar{c}_{\alpha}+\frac{Q_{\alpha}}{\left|z^{a}-z_{(\alpha)}^{a}\right|^{1-d_{\mathrm{Z}}}},
$$

where $\bar{c}_{\alpha}, Q_{\alpha}$ and $z_{(\alpha)}^{a}$ are constants and $d_{\mathrm{Z}}(=0, \ldots, 2)$ is the smeared dimensions in the $\mathrm{Z}$ space. In the case of $d_{\mathrm{Z}}=1$, the power function must be replaced by $\ln \left|z^{a}-z_{(\alpha)}^{a}\right|$. $Q_{\alpha}$ denotes the charge (or mass) of the D4 brane and each D4 brane is located at $z^{a}=z_{(\alpha)}^{a}$.
[1] G. W. Gibbons, H. Lu, and C. N. Pope, Brane Worlds in Collision, Phys. Rev. Lett. 94, 131602 (2005).

[2] W. Chen, Z.-W. Chong, G. W. Gibbons, H. Lu, and C. N. Pope, Horava-Witten stability: eppur si muove, Nucl. Phys. B732, 118 (2006).

[3] H. Kodama and K. Uzawa, Moduli instability in warped compactifications of the type-IIB supergravity, J. High Energy Phys. 07 (2005) 061.

[4] H. Kodama and K. Uzawa, Comments on the fourdimensional effective theory for warped compactification, J. High Energy Phys. 03 (2006) 053.

[5] H. Kodama and K. Uzawa, Moduli instability in warped compactification, arXiv:hep-th/0601100.

[6] P. Binetruy, M. Sasaki, and K. Uzawa, Dynamical D4-D8 and D3-D7 branes in supergravity, Phys. Rev. D 80, 026001 (2009).

[7] P. Binetruy, M. Sasaki, and K. Uzawa, Dynamical solution of supergravity, arXiv:0801.3507.

[8] K. Maeda, N. Ohta, and K. Uzawa, Dynamics of intersecting brane systems-classification and their applications, J. High Energy Phys. 06 (2009) 051.

[9] G. W. Gibbons and K. Maeda, Black Holes in an Expanding Universe, Phys. Rev. Lett. 104, 131101 (2010).

[10] K. Maeda and M. Nozawa, Black hole in the expanding universe from intersecting branes, Phys. Rev. D 81, 044017 (2010).

[11] K. Uzawa, Dynamical intersecting brane solutions of supergravity, AIP Conf. Proc. 1200, 541 (2010).

[12] K. Maeda and M. Nozawa, Black hole in the expanding universe with arbitrary power-law expansion, Phys. Rev. D 81, 124038 (2010).

[13] M. Minamitsuji, N. Ohta, and K. Uzawa, Dynamical solutions in the 3-form field background in the Nishino-Salam-Sezgin model, Phys. Rev. D 81, 126005 (2010).
[14] K. Maeda, M. Minamitsuji, N. Ohta, and K. Uzawa, Dynamical $p$-branes with a cosmological constant, Phys. Rev. D 82, 046007 (2010).

[15] M. Minamitsuji, N. Ohta, and K. Uzawa, Cosmological intersecting brane solutions, Phys. Rev. D 82, 086002 (2010).

[16] K. Uzawa, Cosmological intersecting brane solutions in string theory, J. Phys. Conf. Ser. 259, 012032 (2010).

[17] M. Nozawa and K. Maeda, Cosmological rotating black holes in five-dimensional fake supergravity, Phys. Rev. D 83, 024018 (2011).

[18] M. Minamitsuji and K. Uzawa, Cosmology in p-brane systems, Phys. Rev. D 83, 086002 (2011).

[19] K. Maeda and M. Nozawa, Black hole solutions in string theory, Prog. Theor. Phys. Suppl. 189, 310 (2011).

[20] M. Minamitsuji and K. Uzawa, Dynamics of partially localized brane systems, Phys. Rev. D 84, 126006 (2011).

[21] K. Maeda and K. Uzawa, Dynamical brane with angles: collision of the universes, Phys. Rev. D 85, 086004 (2012).

[22] M. Minamitsuji and K. Uzawa, Cosmological brane systems in warped spacetime, Phys. Rev. D 87, 046010 (2013).

[23] J. Blåbäck, B. Janssen, T. Van Riet, and B. Vercnocke, Fractional branes, warped compactifications, and backreacted orientifold planes, J. High Energy Phys. 10 (2012) 139.

[24] K. Uzawa and K. Yoshida, Dynamical Lifshitz-type solutions and aging phenomena, Phys. Rev. D 87, 106003 (2013).

[25] K. Uzawa and K. Yoshida, Dynamical F-strings intersecting D2-branes in type-IIA supergravity, Phys. Rev. D 88, 066005 (2013).

[26] J. Blåbäck, B. Janssen, T. Van Riet, and B. Vercnocke, BPS domain walls from backreacted orientifolds, J. High Energy Phys. 05 (2014) 040.

[27] K. Uzawa and K. Yoshida, Probe brane dynamics on cosmological brane backgrounds, Phys. Lett. B 738, 493 (2014). 
[28] K. Uzawa, Colliding $p$ branes in the dynamical intersecting brane system, Phys. Rev. D 90, 025024 (2014).

[29] K. Maeda and K. Uzawa, Violation of cosmic censorship in dynamical $p$-brane systems, Phys. Rev. D 93, 044003 (2016).

[30] J. C. Breckenridge, G. Michaud, and R. C. Myers, New angles on D branes, Phys. Rev. D 56, 5172 (1997).

[31] N. Hambli, Comments on Dirichlet branes at angles, Phys. Rev. D 56, 2369 (1997).

[32] V. Balasubramanian, F. Larsen, and R. G. Leigh, Branes at angles and black holes, Phys. Rev. D 57, 3509 (1998).

[33] M. M. Sheikh Jabbari, Classification of different branes at angles, Phys. Lett. B 420, 279 (1998).

[34] D. Youm, Black holes and solitons in string theory, Phys. Rep. 316, 1 (1999).
[35] J. C. Breckenridge, G. Michaud, and R. C. Myers, More D-brane bound states, Phys. Rev. D 55, 6438 (1997).

[36] P. Di Vecchia and A. Liccardo, D branes in string theory, I, NATO Sci. Ser., Ser. C 556, 1 (2000).

[37] P. Di Vecchia and A. Liccardo, D branes in string theory, II., arXiv:hep-th/9912275.

[38] E. Bergshoeff, C. M. Hull, and T. Ortin, Duality in the typeII superstring effective action, Nucl. Phys. B451, 547 (1995).

[39] E. Bergshoeff, R. Kallosh, and T. Ortin, Duality versus supersymmetry and compactification, Phys. Rev. D 51, 3009 (1995).

[40] M. S. Costa and G. Papadopoulos, Superstring dualities and $p$-brane bound states, Nucl. Phys. B510, 217 (1998). 